\title{
MOBILE PHONE APPLICATION TO SUPPORT THE ELDERLY
}

\author{
Maria Panou \\ Center for Research and Technology Hellas \\ L. Mesogion 357-359, Athens, 15231, Athens, Greece \\ mpanou@certh.gr \\ Katerina Touliou \\ Center for Research and Technology Hellas \\ 6th Km Charilaou-Thermis, 57001, Thessaloniki, Greece \\ touliouk@certh.gr \\ Evangelos Bekiaris \\ Center for Research and Technology Hellas \\ L. Mesogion 357-359, Athens, 15231, Athens, Greece \\ abek@certh.gr \\ Taxiarchis Tsaprounis \\ Center for Research and Technology Hellas \\ 6th Km Charilaou-Thermis, 57001, Thessaloniki, Greece \\ taxiarchis.tsaprounis@certh.gr
}

\begin{abstract}
There is a significant increase of the elderly population worldwide, which is accompanied with a rise of the number of people living alone. Moreover, many citizens are at risk due to geographic and social isolation in combination with specific chronic conditions. ICT offers new opportunities in the healthcare area, therefore, within the REMOTE research project, several services have been developed and interconnected
\end{abstract}


(targeted at patients with chronic diseases) available on Symbian OS mobile platforms and computers. This paper presents the Guardian Angel service on the mobile phone, in order to monitor (unobtrusively) the health of the user on the move and alert the medical centre in case of an emergency. The prototype has been evaluated by 30 elderly users and 5 experts, indicating a possible deployment of the application in the healthcare market, but also proposing further improvements for increased usability.

Keywords: Health Monitoring, Elderly, Mobile Application, Chronic Disease, Symbian

\section{INTRODUCTION}

There was a considerable increase in the proportion of persons aged 65 or over during the 20 year period from 1990 to 2010 . Worldwide, the proportion of people aging 65-79 years old increased from 5.1\% in 1990 to $6.1 \%$ in 2010, while those aged 80+ increased from $1.1 \%$ to $1.5 \%$. The on-going ageing process is most pronounced in Europe, Latin America and the Caribbean, Asia and Oceania (Heilig, 2011). An ageing population is expected to result in additional demands for a range of health and health-related products and services. The medical monitoring and care in rural areas of elderly individuals having chronic illnesses and/or disabilities frequently presents a problem for care personnel, physicians and service providers. Elderly and disabled individuals often suffer marginal health care monitoring and medical services due to geographical and social isolation. Information and Communication Technologies (ICT) solutions for the elderly are becoming more commonly used in many ways to improve the living experience and quality of life of people at home. Moreover, the percentage of the ageing population using ICT is also rising annually (Nugent, 2007).

REMOTE was a research project co-funded by the European Commission (under the Ambient Assisted Living joint program), which aimed at defining and establishing a multidisciplinary and integrated approach to ICT for addressing real needs of elderly citizens at risk due to geographic and social isolation, in combination with specific chronic conditions and the coexistence of lifestyle risk factors, such as obesity, blood pressure, poor eating/drinking habits, stress, etc. Several applications have been integrated on mobile and portable platforms to ensure remote access to various services related to the independent living and support of the elderly. One of them is the Guardian Angel application which aims to support the users on the move via monitoring health 
parameters based on state-of-the-art wearable and mobile systems.

\section{THE GUARDIAN ANGEL APPLICATION}

The system design was based on the real needs of stakeholders (formal and informal care-givers, health care and emergency support service providers, elderly associations, representatives of the scientific community, etc.) and requirements for the elderly (facing chronic conditions, e.g. stroke, asthma, hypertension, etc.). This led to the definition of REMOTE use cases, along with all the necessary details for the system functionality and output for the benefit of the end-users (Bekiaris \& Gkaitatzi, 2010). These use cases formed the basis for the definition of the system requirements and specifications that were fed to the developers' team.

The Guardian Angel Mobile Application is developed for Symbian OS platforms. Devices based on Symbian are a big market, as they accounted for $43.5 \%$ of worldwide smart phone sales in 2010. Sensor-enhanced devices are incorporated for the unobtrusive monitoring of various vital parameters such as the heart rate and ECG, breathing rate, posture and activity, skin temperature, blood pressure, weight, etc. Appropriate alerts can be generated so as to assist the patients in avoiding or overcoming hazardous health conditions (e.g. arrhythmia, high blood pressure, falls). The sensor-enhanced devices can communicate via Bluetooth with the patient's mobile device, forming in this way a body sensor network around the user for health monitoring purposes. Screenshots of the Guardian Angel application follow below.
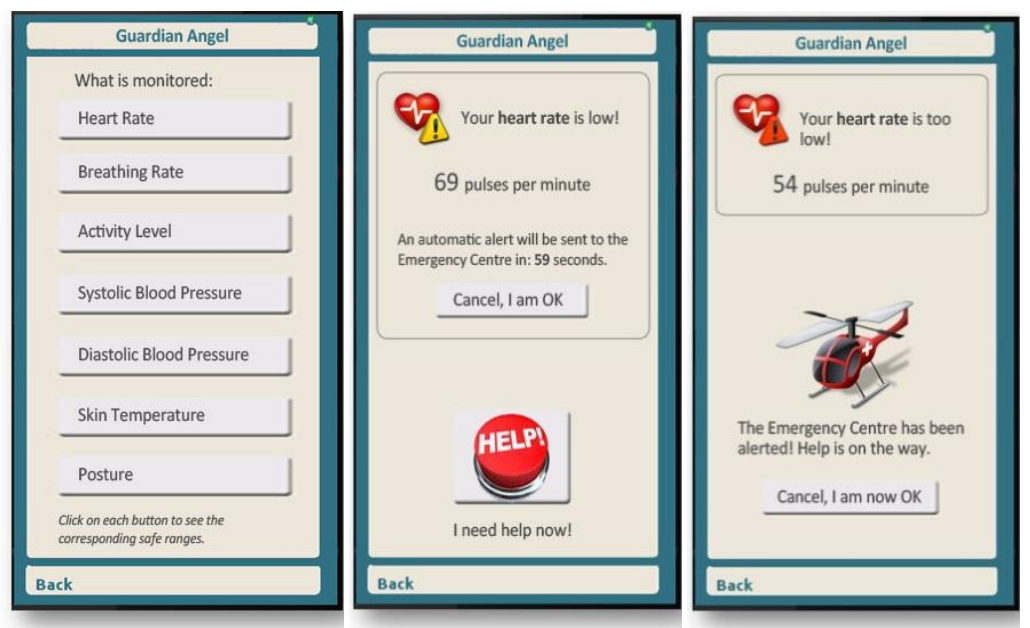

Figure 1 Screenshots of the Guardian Angel Application 
In case of an alert, the user is notified and an orange alert is transmitted to the emergency service of the Medical Center (Triantafyllidis, Koutkias, Chouvarda, \& Maglaveras, 2013). If the alert persists for a period of 55 seconds, a red alert is triggered and sent to the Medical Center in order to take appropriate actions. The patient is also notified in case his/her monitoring parameters return back to safe levels. The patient's monitoring values (received every second) are sent to the back-end system periodically, every 2 minutes, so that the health professionals can be informed of his/her current health status. Moreover, the patient is informed in case internet connection is lost. User interface adaptation is supported with color and language settings.

\section{SYSTEM EVALUATION}

The proposed Guardian Angel application was firstly tested by 5 experts, when the application was not at its final stage, in order to examine easiness of use and identify potential areas of difficulties and errors and propose improvements. More specifically, they interacted with the functions and interfaces of the software/hardware. Real life scenarios (test cases) were used to examine whether an average user could follow the process and complete all the possible tasks efficiently and effectively. An overall positive feedback was given by the experts, as nearly all agreed that the information presented on the screen was simple. While most of them seemed to be fully satisfied, or at least to some extent, with issues of data privacy, system personalization and consistency of users' actions to system output, a few concerns were noted on the clarity of information and system actions visibility. The areas requiring further work concern the support of the system in case of error messages, with clear explanations provided to the user on the problem and on what is expected of him/her.

An updated, mature version of the application (taking into account the experts' feedback) was then tested by 30 elderly users (with various chronic diseases). Evaluation was based on completion of specific tasks (scenarios). The users had the REMOTE system installed on their mobile phones. Calculation of task completion success rates was based on both users' and facilitators' ratings. Users had to state if they thought they successfully completed each task and at the same time the facilitators recorded their own rating. The average score from both facilitators and user gave the overall user success rate for the Guardian Angel application, which reached 97.5\%. This is quite impressive taking into account that most users (83\%) were not acquainted with touch screen mobile phones. 
The helpfulness of the application in their daily lives and the consistency of information were rated positively. Users were comfortable with using the system, however, since older people are used to receiving health related information from their physicians, they suggested that training into the terminology of health indicators presented on the screen is important (and thus increase willingness to use and confidence). Patients often suggested that long-term use was necessary for them to be sure of the benefits of this application. Overall, users stated that they were enthusiastic about the potential of using such applications and did not worry about personal data monitoring by the system. On the contrary, most of them (76\%) stated that they would like to be monitored in order to feel safer knowing that in case of emergency-or if their vital signs were critical-an alert would be sent to both their appointed (either formal or informal) carers and health care professionals.

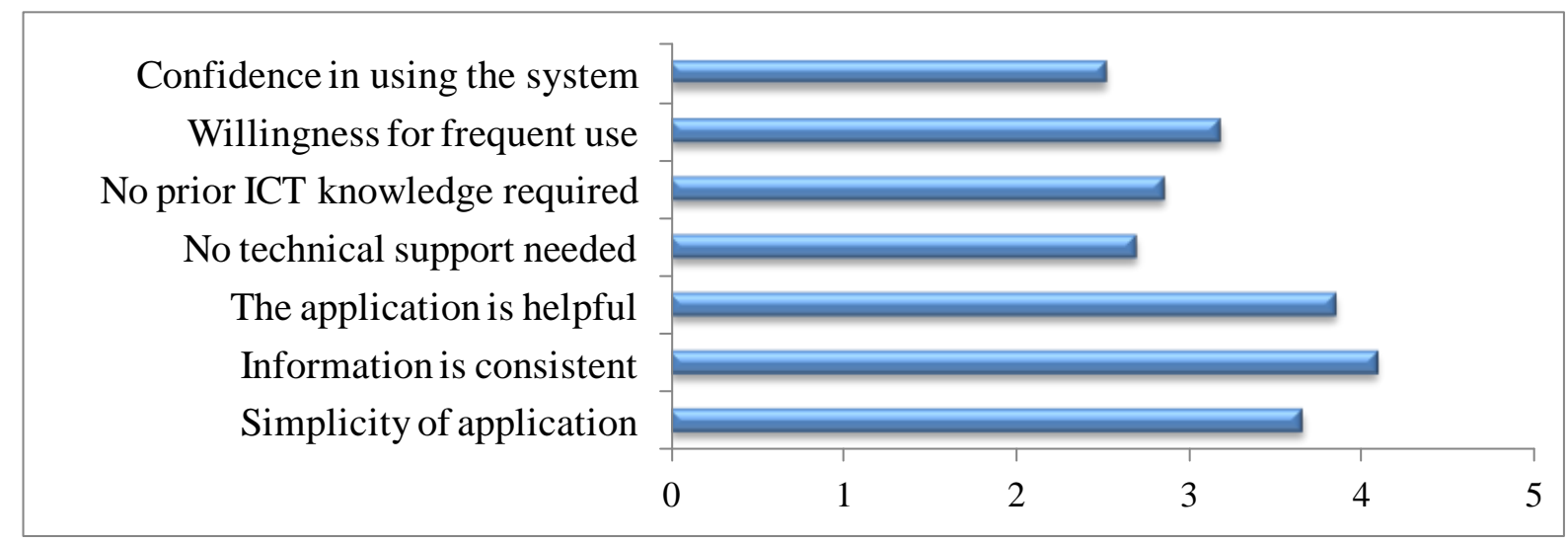

Figure 2 Mean Scores for the Guardian Angel Application Evaluation (Scaling Explanation: from 1 'Totally Disagree', to 5 'Totally Agree')

The usability score reached $67.3 \%$, well above average, but also indicating that further improvements could be made, resulting in an even more usable system. Therefore, decreasing complexity and amount of information displayed on the screen might increase usability. Finally, the addition of a help menu (i.e. like a virtual assistance for each step/action) would probably increase usability ratings. 


\section{CONCLUSION}

The presented Guardian Angel application for elderly people was based on a philosophy of consistent involvement of users. Evaluation findings support the increased potential of the application for deployment and penetration into existing telemedicine and health mobile services. This philosophy is going beyond the utility-focus so far, as it is not limited to the main user group of the elderly, but applies to all target actors (formal/informal care givers, social service providers, family, friends). A promising market opportunity is foreseen, as $1.4 \mathrm{bn}$ smartphone holders are expected to use mobile health systems in 5-year time (Global Mobile Health Market Report, 2010-15). Developing countries are also expected to benefit from the Guardian Angel application, as REMOTE offers easily manageable services for a wide spectrum of socioeconomic environments (not only rich ones), with various social and healthcare services and systems.

\section{REFERENCES}

Bekiaris, E., \& Gkaitatzi, O. (2010, May). Use cases and scenarios for remote support of the elderly to live autonomously alone. Presented at IST Africa Conference, Durban, South Africa.

Global Mobile Health Market (GMHM) Report (2010-2015). Retrieved on November 10, 2010 , from http://www.research2guidance.com/500m-people-will-be-using-healthcare-mobile-a pplications-in-2015/

Heilig, G.K. (2011, November). World population prospects: The 2010 revision. United Nations, Department of Economic \& Social Affairs, International seminar on Population Estimates and Projections.

Nugent, C.D. (2007). ICT in the elderly and dementia. Aging \& Mental Health, 11(5), 473-476. http://dx.doi.org/10.1080/13607860701643071.

Triantafyllidis, A., Koutkias, V., Chouvarda, I., \& Maglaveras, N. (2013). A pervasive health system integrating patient monitoring, status logging, and social sharing. IEEE Journal of Biomedical and Health Informatics, 17(1), 30-37. http://dx.doi.org/10.1109/TITB.2012.2227269. 